\title{
Fact-checking en el periodismo mexicano: Análisis de la experiencia Verificado 2018
}

\author{
Fact-checking in Mexican journalism: \\ Analysis of Verificado 2018 experience
}

Rocío Galarza-Molina (Dhttp://orcid.org/0000-0001-7002-0638

Universidad Autónoma de NuevoLeón, México,rociogalarzamolina@gmail.com

\begin{abstract}
This paper analyzes how fact-checking is incorporated into Mexican journalism taking into account the experience of Verificado 2018. In-depth interviews were conducted with journalists who collaborated in the project. The results indicate that Verificado 2018 journalists conceive fact-checking as a part of traditional journalism and its democratic objective. However, they pointed out failures in the practice of journalism in Mexico and threats due to the growing phenomenon of fake news. In this context, Verificado 2018 opted for alternatives in the performance of its fact-checking tasks, such as a more horizontal relationship with sources, interaction with the public, collaborative journalism and media literacy stories. They considered though that Verificado 2018 does not have an activist mission. Verificado 2018 fueled the discussion on the quality of Mexican journalism and how journalists can contribute to its improvement.
\end{abstract}

Key words: fact-checking, journalism, Verificado 2018.

Resumen: Este trabajo analiza cómo se incorpora el fact-checking en el periodismo mexicano, tomando en cuenta la experiencia de Verificado 2018. Se realizaron entrevistas a profundidad con periodistas que colaboraron en el proyecto. Los resultados indican que los periodistas de Verificado 2018 conciben al fact-checking como parte del periodismo tradicional y su objetivo democrático. No obstante, señalaron fallas en la práctica del periodismo en México y amenazas por el creciente fenómeno de noticias falsas. En este contexto, Verificado 2018 apostó por alternativas en el desempeño de sus labores de fact-checking, como una relación más horizontal con fuentes, interacción con el público, periodismo colaborativo y notas de educación mediática. Empero, consideraron que Verificado 2018 no tuvo una misión activista. Verificado 2018 avivó la discusión sobre la calidad del periodismo mexicano y de qué forma periodistas pueden contribuir a su mejora. Palabras clave: fact-checking, periodismo, Verificado 2018. 


\section{Introducción}

El auge de Internet y de las redes sociales ha favorecido la difusión de información acerca de asuntos públicos. Los mensajes a través de tales canales se difunden muy rápido y tienen el potencial de alcanzar viralidad. Además, al democratizarse el acceso a estos medios existen menos filtros para difundir información, por lo que esta no siempre es veraz. Estos dos factores han facilitado la explosión del fenómeno de fake news, artículos noticiosos que son intencional y verificablemente falsos y que pueden engañar al público (Allcott y Gentzkow, 2017: 4).

En el ámbito político, estas noticias falsas pueden tener ramificaciones normativas peligrosas, puesto que los votantes no solo están desinformados sino malinformados (Kuklinski et al., 2000: 792). Ante un clima informativo viciado por la presencia de múltiple información y noticias intencionalmente falsas, el fact-checking ha cobrado fuerza como una herramienta para corroborar o desmentir información y verificar el discurso público.

Esta investigación estudia la experiencia de uno de los primeros esfuerzos por hacer fact-checking de manera sistemática en México, surgido en el contexto de la elección presidencial de 2018. Verificado 2018 fue un proyecto de más de 80 aliados, entre ellos medios, universidades y organizaciones civiles. Su objetivo era "verificar, en tiempo real, información relevante sobre el proceso electoral” (Khatib y Larrea, 2018), surgida del discurso de actores públicos o notas falsas. El proyecto inédito reunió a medios digitales y tradicionales con diversas tendencias editoriales.

Este trabajo analiza cómo se incorpora el fact-checking en el periodismo mexicano tomando en cuenta la experiencia de Verificado 2018. Se realizaron entrevistas a profundidad con periodistas que colaboraron en el proyecto. Específicamente, el interés del presente trabajo fue conocer la perspectiva de estos periodistas respecto a cómo conciben al fact-checking, cómo difiere del periodismo tradicional y cuál fue el impacto del proyecto.

Los resultados indican que los periodistas de Verificado 2018 consideran al fact-checking como parte del periodismo tradicional y su objetivo democrático. No obstante, señalaron fallas en la práctica del periodismo en México y amenazas por el creciente fenómeno de noticias falsas. En este contexto, Verificado 2018 apostó por alternativas en el desempeño de sus labores de fact-checking, como una relación más horizontal con fuentes, interacción con el público, periodismo colaborativo y notas de educación mediática. Empero, los entrevistados consideraron que el proyecto no tenía una misión activista. En la conclusión se discute cómo Verificado 2018 avivó la 
discusión sobre la calidad del periodismo mexicano y de qué forma los periodistas pueden contribuir a su mejora, así como los límites derivados del arraigo a los valores de la profesión.

\section{Revisión de literatura}

¿Qué es el fact-checking?

Definición y antecedentes

El fact-checking consiste en evaluar, de manera sistemática, la veracidad de las aseveraciones de políticos y de información noticiosa que pueda impactar en la opinión pública (Graves, 2018: 615). Para Amazeen (2017: 3), el fact-checking político tiene tres objetivos fundamentales: informar al público, mejorar el comportamiento político y mejorar el periodismo. Fue en 2003 en Estados Unidos cuando emergió la primera organización dedicada precisamente a esta misión, Factcheck.org. En los últimos años, los esfuerzos de fact-checking han adquirido prominencia no solo en Estados Unidos, sino alrededor del mundo. Tal éxito se debe parcialmente a que estas propuestas surgen en momentos de alta desconfianza en los actores públicos (políticos y medios) y en un contexto de comunicación masiva individualizada donde la digitalización ha cambiado rutinas y técnicas de producción de contenido informativo (López et al., 2016: 2). Asimismo, esta expansión se ha visto favorecida por la oportunidad que brindan las redes sociales para distribuir y viralizar el contenido verificado (Echeverría, 2017: 11).

Existen varios aspectos a considerar al hablar de fact-checking. Si bien, los periodistas siguen ciertos criterios de verificación de datos de manera interna antes de publicar cierta información para eliminar posibles errores, la forma de periodismo conocida como fact-checking se refiere a la actividad en la cual se publicitan declaraciones que han sido determinadas como incorrectas (Amazeen, 2017: 3). Además, los verificadores de información deben estar libres de tendencias partidistas o ideológicas en su práctica, o de lo contrario se trata de actores políticos (Graves, 2018: 616). En Estados Unidos, el fact-checking suele estar en manos de periodistas; sin embargo, en otras partes del mundo, activistas o académicos se involucran también en estas labores (Cheruiyot y Ferrer-Conill, 2018: 965; Mena, 2018: 4).

Los efectos del fact-checking en las creencias o actitudes de las personas son mixtos, pues algunos individuos se aferran a percepciones erróneas a pesar de encontrarse con correcciones (Amazeen, 2017: 3). Algunos estudios han hallado que el fact-checking tiene efectos positivos moderados en 
la corrección de información errónea (Walter y Murphy, 2018: 432); tales efectos van desde influir en la percepción de la información que se presenta en propaganda política (Fridkin et al., 2015: 127), en la evaluación de candidatos políticos (Wintersieck, 2017: 304) y en el nivel de conocimiento sobre política y posturas políticas de candidatos (Gottfried et al., 2013: 1558).

Por otro lado, Amazeen (2015) apunta que el impacto de la verificación se observa sobre todo en el discurso político, ya que los políticos dicen menos falsedades en sus discursos al saberse monitoreados. Igualmente, el fact-checking puede tener impacto en la confianza del público en los medios (Pingree et al., 2018: 1).

\section{Fact-checking y periodismo}

La emergencia del fact-checking ha sido explicada como un movimiento reformista profesional por parte de la misma comunidad de periodistas (Graves, 2013: 1). Graves y Konieczna (2015: 1977) encuentran que los proyectos de fact-checking reflejan una crítica al periodismo político convencional que solo transmite declaraciones, apelando así a la misión democrática del periodismo para enfocarse en formas en las cuales se pueden atender fallas y amenazas en la profesión. Investigaciones previas han señalado que periodistas perciben que el fact-checking es congruente con los ideales tradicionales del periodismo y que esta vinculación motiva a los periodistas a realizar este tipo de labor (Graves et al., 2016: 102).

Pese a diferencias en los procedimientos para ejecutar esta actividad, existe amplio acuerdo entre fact-checkers acerca del principal propósito del fact-checking: informar al público (Amazeen, 2013: 5). Concretamente, los periodistas consideran que los objetivos de esta actividad son evaluar la veracidad de declaraciones hechas por personajes públicos y desmentir noticias falsas (Mena, 2018: 8). No obstante, los periodistas que participan de esta labor (al menos en Estados Unidos) han expresado su desacuerdo respecto a que el fact-checking tiene una misión de promover una causa social, distanciándose así de un fin activista (Mena, 2018: 9).

Por otra parte, algunos periodistas también han compartido su perspectiva respecto al impacto del fact-checking. Un aspecto importante a considerar es cómo impacta el fact-checking al periodismo. Una investigación de Brandtzaeg et al. (2018: 1118) encontró que los periodistas valoran el potencial de los servicios de fact-checking para sensibilizarse sobre hechos que necesitan ser verificados.

De manera más amplia, el fact-checking también puede influir en el periodismo para alejarse de un formato más tradicional de "él dijo-ella dijo" 
e introducir un modelo sistemático de fact-checking (Amazeen, 2013: 14). Asimismo, el fact-checking también se caracteriza por un énfasis en prácticas que involucran el análisis de datos (Cheruiyot y Ferrer-Conill, 2018: 965). Respecto a un impacto más allá del periodismo, Amazeen (2013: 8) señala que los fact-checkers tienen pocos elementos para determinar de qué forma influyen en el público y se basan más en intuición y anécdotas. Sin embargo, entrevistas realizadas con fact-checkers revelaron que estos señalan como consecuencias de su trabajo: mayor disponibilidad de información para el público y cambios en la retórica de los políticos (Amazeen, 2013: 12).

Si bien los periodistas no cuentan con herramientas para medir el impacto del fact-checking de forma precisa, sus percepciones sobre los efectos que tienen resultan relevantes, porque de estas podrían depender sus motivaciones para participar en proyectos de fact-checking o incorporar secciones de esta naturaleza en sus medios.

Verificado 2018 y el contexto mexicano

Antes de Verificado 2018, en México existían pocos esfuerzos de fact-checking en el periodismo o fuera de él. Un censo de iniciativas de fact-checking en español (Vizoso y Vázquez-Herrero, 2019: 138) identifica en México solo dos proyectos de este tipo: El Sabueso de Animal Politico, que opera desde enero de 2015, y El Polígrafo de Milenio, que actualmente está inactivo. Esto pese a que el fact-checking de declaraciones políticas es de particular importancia en México, debido a la historia de coerción periodística y el clientelismo político que prevalece en el sistema de medios en el país (Martínez-Carrillo y Tamul, 2019: 2598). Ante tal contexto, la confianza ciudadana en medios mexicanos previo a la elección de 2018 era baja y las noticias falsas eran identificadas como un potencial riesgo para la toma de decisiones en el país (Edelman Trust Barometer, 2018). Además, durante elecciones anteriores se habían identificado esfuerzos por manipular la opinión pública en redes sociales a través de compra de bots (Martínez, 2018) y propaganda política disfrazada de opiniones de celebridades en Twitter (Redacción Animal Político, 2015). Estas condiciones, aunadas a la tendencia mundial de iniciativas de fact-checking, apuntalaron el nacimiento de Verificado 2018.

El 1 de julio de 2018 en México se llevaron a cabo elecciones en las que se votó al presidente de la República, 128 senadores y 500 diputados; además, se celebraron elecciones estatales y locales en 30 estados del país. Verificado 2018 operó del 12 de marzo al 5 de julio de 2018, con el objetivo de "combatir las noticias falsas y denunciar el uso tramposo de la información” (Verificado 
2018, 2018a) durante la precampaña y la campaña electoral. La iniciativa fue dirigida por los medios Animal Político y AJ Plus en Español, así como por la organización Pop Up Newsroom, dedicada a apoyar al periodismo colaborativo. El proyecto contó con un total de 80 aliados, entre ellos importantes medios mexicanos, universidades y organizaciones no gubernamentales.

Verificado 2018 realizó más de 400 notas y 50 videos. Las notas consistían en verificaciones del discurso público, por ejemplo, la nota respecto a las declaraciones del candidato del Partido Morena titulada "Inexactos, los cálculos de López Obrador para medir la penetración de la telefonía móvil” (Díaz, 2018); y verificaciones de notas falsas, por ejemplo, la que desmentía un rumor circulado en redes sociales respecto al candidato del PAN, titulada "Los falsos lazos familiares entre Ricardo Anaya y los Salinas” (De la Torre, 2018).

Sus videos tenían contenido educativo acerca de cómo verificar información o informaban sobre cómo funcionan las campañas digitales de desinformación (Verificado 2018a y 2018b). Además, se hicieron verificaciones en tiempo real de los debates presidenciales. Estas publicaciones atrajeron alrededor de cinco millones de visitas a su portal de Internet y algunos de sus videos alcanzaron hasta un millón de visitas (Verificado 2018, $2018 \mathrm{~b}$ ). Las verificaciones se distribuyeron a través de su sitio de Internet y de las redes sociales de Verificado 2018, que en cuestión de meses llegaron a tener más de 200 mil seguidores en Facebook y Twitter, lo cual resulta significativo si se observa que sitios consolidados durante años como Factcheck. org cuentan con 157 mil seguidores en Twitter (Martínez-Carrillo y Tamul, 2019: 2599). El aporte de contenido atractivo diseñado para compartirse en redes y la alta interacción e inclusión de la audiencia son considerados como las claves para el éxito de las plataformas del proyecto (Flueckiger, 2019). En vista de estos resultados, Verificado 2018 es considerado un ejemplo de periodismo colaborativo a nivel mundial, lo cual lo posiciona como un caso para imitar y estudiar en muchos países (Magallón, 2019: 259).

Tomando en cuenta la caracterización del fact-checking como un movimiento reformista de la profesión, con el fin de explorar cómo se incorpora Verificado 2018 en el contexto mexicano, se establecieron las siguientes preguntas de investigación.

PI 1. De acuerdo con los participantes de Verificado 2018, ¿cómo conceptualizan el trabajo de fact-checking realizado en el proyecto y qué relación tiene este con el periodismo tradicional?

PI 2. De acuerdo con los participantes del proyecto, ¿existía un componente de activismo en la labor realizada por Verificado 2018?

PI 3. Según la percepción de los integrantes del proyecto, ¿cuál es el impacto que tuvo Verificado 2018? 


\section{Método}

La entrevista provee la posibilidad de adentrarse en la perspectiva de otra persona, permitiendo al sujeto explicar sus experiencias en sus propios términos (Patton, 1990). De tal forma, este método permite profundizar sobre las ideas, pensamientos, opiniones, actitudes y motivaciones de otros (Berger, 2000: 113). Para responder a las preguntas de investigación planteadas se entrevistó a actores vinculados con Verificado 2018 para conocer más a fondo las motivaciones y las prácticas de quienes estuvieron involucrados en el proyecto. Entre noviembre de 2018 y enero de 2019 se efectuaron 13 entrevistas a profundidad semiestructuradas vía telefónica o por Skype de entre 25 y 65 minutos. Los entrevistados ( 6 del sexo masculino y 7 del sexo femenino) fueron periodistas que desempeñaron distintas funciones en el proyecto Verificado 2018. La Tabla $1^{1}$ especifica quiénes fueron las personas entrevistadas y cuál fue su rol en el proyecto.

Se estableció un protocolo de entrevista con algunos temas y preguntas a seguir durante la interacción, como, por ejemplo, razones por las que surgió Verificado 2018, diferencias que existen con el periodismo tradicional y alcances del proyecto. Las entrevistas fueron transcritas en su totalidad y se realizó un análisis temático a partir de las respuestas de los participantes. El análisis temático es un proceso para codificar información cualitativa, con el objetivo de identificar temas a partir de datos (Boyatzis, 1998: vi). Para determinar dichos temas se aplicaron procedimientos del método comparativo constante, es decir, se redujo la información a partir de codificar y recodificar hasta alcanzar la saturación teórica (Glaser y Strauss, 1967: 111).

\section{Resultados}

\section{Objetivos de Verificado 2018 y relación con el periodismo tradicional}

Fact-checking: Periodismo 101

$\mathrm{Al}$ referirse a cómo conciben el fact-checking, las respuestas de los integrantes de Verificado 2018 coincidieron con la tendencia de fact-checkers a nivel mundial, relacionando esta actividad con el deber ser tradicional del periodismo. Los entrevistados argumentaron que esta actividad es un elemento clave en todo tipo de periodismo, llamándolo "lo más básico del periodismo" (De la Torre, comunicación personal, 8 de enero de 2019) o "periodismo 1 Esta tabla se encuentra en el Anexo, al final del presente artículo (Nota del editor). 
puro" (Plascencia, comunicación personal, 29 de noviembre de 2018), enfatizando que su ejecución no implicaba una técnica distinta a lo que hace cualquier periodista al publicar información (Montalvo, comunicación personal, 13 de diciembre de 2018). Es decir, desde su perspectiva, revisar la veracidad de la información que se publica es un deber de todo periodista. Además, enfatizaron que al realizar esta actividad se guiaban por el principio de objetividad, piedra angular del periodismo moderno (Muñoz-Torres, 2012: 566). Esto es, los entrevistados apuntaron que en las verificaciones mantenían como prioridad ser neutrales y revisar todo tipo de información, sin importar posturas ideológicas.

Al expresarse respecto a los objetivos de esta actividad, los entrevistados reiteraron la misión del proyecto de combatir la desinformación. Señalaron el propósito de informar al ciudadano y de esta manera ayudarle a tomar decisiones en la elección. Así lo explica Diego de la Mora, Coordinador de Metodología y Datos en el proyecto.

El objetivo final era que quien llegara a la casilla hubiera tomado la decisión de por quién votar con mayor cantidad de información cierta posible, con libertad de votar por quien decidiera y no influido por noticias falsas (De la Mora, comunicación personal, 9 de enero de 2019).

Es así que las motivaciones para hacer fact-checking en Verificado 2018 están vinculadas con un objetivo tradicional que define al periodismo: la intención de proveer información para un público capaz de autogobernarse (George, 2013: 493). La práctica de esta actividad no se entiende como fuera de las labores tradicionales de dicha profesión.

\section{Diferencias con periodismo convencional}

Pese a que identificaron objetivos consistentes con los ideales del periodismo, los entrevistados sí reconocieron que había diferencias entre el periodismo de Verificado 2018 y el que se practica típicamente en México. Por un lado, los entrevistados mencionaron diferencias de logística en la forma de trabajar, de entrada por que no hacían notas del día. Su trabajo difería también, pues contaban con poco tiempo para hacer notas que requerían análisis detallados y énfasis en datos (por lo que incluso a algunos colaboradores los llamaban "dateros"). Otra diferencia en la forma de trabajar fue la relación que se mantenía con otros colaboradores de Verificado 2018, organizaciones no gubernamentales y académicos que fungieron como fuentes expertas. Aunque estos colaboradores comúnmente contribuyeron como fuentes de manera tradicional, en ocasiones también escribieron notas en conjunto con reporteros. Así lo explica la reportera Ana Karen De la Torre. 
Por ejemplo con Oxfam a mí me tocó hacer una verificación y era como de rebotarnos el texto, ¿no? Ellos hacían la propuesta y yo la editaba, y luego se las pasaba y así estuvo como rebotando [...] Y también los debates, ahí estaban con nosotros (De la Torre, comunicación personal, 8 de enero de 2019).

La relación con el público también fue distinta, pues si bien la mayoría de los medios tienen interacción con su audiencia en la actualidad, Verificado 2018 puso especial atención a esta comunicación, manteniendo a sus community managers al tanto de las peticiones de verificaciones que llegaban a través de redes y abriendo una línea a través de WhatsApp para ese mismo propósito. Esta estrategia de WhatsApp en particular fue significativa, ya que se estableció una relación de uno a uno con los lectores más fieles.

Por otro lado, los periodistas mencionaron diferencias derivadas de las deficiencias del periodismo que se practica comúnmente en México. Aldo Nicolai, reportero de Verificado 2018, lamentó la falta de rigor y verificación adicional en ciertas expresiones del periodismo mexicano.

No quiero generalizar, porque obviamente que hay todo tipo de redacciones en México pero creo que sí a muchas les hace falta, en el periodismo normal, el rigor un poco del fact-checking, ya sea una crónica, un reportaje, lo que sea. A veces te sueltan las cifras, los periodistas las toman, las dan por bien vistas porque las dice equis diputado, equis gobernador y no se dan a la tarea de corroborar si esas cifras en realidad son ciertas (Nicolai, comunicación personal, 13 de enero de 2019).

De tal manera, los entrevistados coincidieron en que aunque el factchecking debería estar presente en todo tipo de periodismo, en muchas ocasiones no lo está, por falta de tiempo, bajos sueldos, poco personal o por falta de especialización en ciertas fuentes. Los entrevistados mantuvieron una postura cautelosa al referirse al trabajo de otros periodistas, argumentando que no podían generalizar respecto a estos problemas en la calidad del trabajo que realizaban en otros medios y subrayando que no les correspondía a ellos dar clases sobre cómo hacer periodismo.

Igualmente, destaca que Verificado 2018 no realizaba verificaciones de notas de otros medios, pues la intención, según afirman, era verificar información, no medios. De tal forma, cuando se trataba de medios aliados, cualquier problema identificado con la información de una nota se manejaba de manera interna, y solamente denunciaban y verificaban páginas de Facebook que generaban noticias intencionalmente falsas para engañar a la población.

Esta postura respecto a otros medios cobra sentido si se considera que servicios de fact-checking y verificación operan en simbiosis con medios tradicionales, quienes promueven su trabajo (Graves, 2013: 257), tal y como ocurrió en el caso de Verificado 2018 que se valía de medios aliados para difundir la información que producía. 


\section{Educación mediática y límites con activismo}

En la mayoría de las notas de Verificado 2018 se hacía verificación de discurso político o bien se desmentían notas falsas. Sin embargo, algunas notas -a las que los entrevistados llamaban explainers o explicadores- presentaban información básica sobre las elecciones, como, por ejemplo, aspectos referentes a las credenciales de elector o sobre cómo ejercer el derecho al voto.

Además, Verificado 2018 publicaba otras notas a la que los periodistas entrevistados denominaban de media literacy (alfabetización mediática) a cargo de los reporteros de AJ Plus. De los 66 videos producidos por Verificado 2018 que están publicados en sus cuentas de redes sociales, alrededor de 24 presentan contenido que directamente hace referencia a problemas que impactan la información disponible en redes sociales, como los fenómenos de noticias falsas, uso de bots en campañas políticas, manipulación de imágenes y videos, o bien, otros videos que contienen tutoriales para que los lectores revisen por sí mismos la información que reciben y comparten en redes.

Hay que tomar en cuenta que no toda la gente tiene acceso a las mismas herramientas, entonces esto [estas notas] era un poco una manera de tratar de acercar a la gente a esas herramientas que están muy a la mano y que puedes usar, con tu propio celular puedes hacer una verificación [...] O sea, un poco para que la gente también pudiera hacerlo ellos mismos, y así si veían una información, empezaran a dudar y naciera este sentido crítico ¿no? a la hora de consumir información (Plascencia, comunicación personal, 29 de noviembre de 2018).

Los periodistas de Verificado 2018 insistían en la necesidad de información de esa naturaleza para que el ciudadano reflexione críticamente sobre las noticias e información que consumen y esté al tanto de las consecuencias de compartir información falsa, en un contexto en el que prevalece la desinformación.

El problema de la desinformación es algo con lo que vamos a tener que convivir ya en el día a día, o sea, es un tema que ya empezó hace unos años, pero no va a terminar. Es muy fácil de hacerlo [las notas desinformativas], es muy efectivo, entonces es importante enseñarle a la gente a discernir lo que es cierto, lo que es falso (Escamilla, comunicación personal, 19 de diciembre de 2018).

Las explicaciones que los periodistas daban acerca de la necesidad de este tipo de notas educativas son consistentes con la definición de educación mediática -la habilidad de tener acceso a medios y evaluarlos (Media Literacy Project, 2015)-, concepto que ha ganado atención por la necesidad de ayudar a la gente a protegerse a sí misma frente a los múltiples posibles efectos negativos que existen del consumo de medios (Potter, 2010). 
Sabíamos que Verificado no iba a ser para siempre, entonces decíamos, es que qué va a pasar, o mucha gente decía, qué va a pasar después, qué vamos a hacer después de que ya no estén, las noticias falsas van a seguir [...] Tenemos que nosotros también tener esta cultura cívica entre comillas de verificar la información que vemos en Internet (García, comunicación personal, 19 de diciembre de 2018).

No obstante, la mayoría de los entrevistados no alinearon estos esfuerzos con una causa activista, sino que la consideraban como parte del periodismo. Tania Montalvo lo explicó de la siguiente forma:

No creo que sea un tema de activismo, o sea lo que estamos haciendo es periodismo, te decía que yo no creo que nada de lo que hacemos en Verificado sea distinto a lo que tendríamos que hacer como periodistas todos los días [...] Lo que estamos haciendo como periodistas es darle a la gente herramientas para que tenga una participación pública informada, activa o como desee tenerla (Montalvo, comunicación personal, 13 de diciembre de 2018).

\section{Impacto de Verificado}

Consecuencias para la opinión pública y la retórica política

Los integrantes de Verificado 2018 se manifestaron satisfechos por el trabajo realizado, al reconocer que, desde su perspectiva, el proyecto tuvo consecuencias positivas en la opinión pública y en el desarrollo de la retórica política, cumpliendo de tal manera con el objetivo democrático tradicional del periodismo. Una consecuencia importante fue que Verificado 2018 logró posicionarse, de manera rápida, como un actor más que figuraba como parte del debate público durante el periodo electoral y que era referente para el público.

Un ejemplo que varios mencionaron como un indicador de la magnitud de su trabajo fue cuando después del primer debate presidencial, una nota en la que se verificaban cifras brindadas por el candidato del Partido Acción Nacional (PAN), Ricardo Anaya, adquirió viralidad y se generó el hashtag \#mientocomoAnaya.

Los entrevistados consideraron que Verificado 2018 se convirtió en el fiel de la balanza al cual el público podía acudir si había discusiones sobre la información de las elecciones. Además de que la ciudadanía los tomaba en cuenta para verificar la información, también ganaron la atención de por lo menos algunos actores políticos importantes, quienes les enviaban información detallada sobre las fuentes de los datos que utilizaban y modificaron su retórica al saber que serían monitoreados por Verificado 2018. 
[En elecciones pasadas] podían gozar en su discurso de completa impunidad y ahora no, este ejercicio sí los hizo como darse cuenta de que tienen que ser más cuidadosos de lo que decían, que hacer difamaciones, $o$ acusaciones falsas, no iba a ser un camino tan fácil (Ávila, comunicación personal, 17 de diciembre de 2018).

Consecuencias para el periodismo

De acuerdo con los periodistas, Verificado 2018 también tuvo impacto en el periodismo mexicano. Por un lado, los entrevistados argumentaron que esta iniciativa contribuyó a generar debate sobre la calidad del periodismo en el país. Así, estas conversaciones podrían llevar a recordar la misión original del periodismo que va más allá del aspecto comercial, pues existe una labor social al practicar esta profesión.

Asimismo, además de revisitar los objetivos del periodismo, este debate sobre calidad podría retomar los elementos clave para alcanzar esta calidad, como la corroboración de información y la transparencia en cómo se obtiene la misma.

Otro importante impacto de Verificado 2018 fue que demostró la necesidad y el potencial de proyectos de periodismo colaborativo en el país. Los entrevistados recalcaron que el éxito del proyecto fue en parte por haber sido un esfuerzo colectivo de un gran grupo de medios y organizaciones académicas y civiles. Montserrat Maldonado, reportera de Verificado 2018, explicó de qué forma contribuían los medios aliados.

Los aliados nos servían para saber cómo estaba cada estado de primera mano [...] Esta distribución de medios sí te ayuda a cubrir mejor el panorama electoral y el periodismo colaborativo es algo en lo que cree mucho Animal [Político] de que vamos todos juntos y no solamente yo me voy a pelear por la nota y no te la voy a dar, sino que fue un equipo muy, muy grande. Yo creo también por eso tuvo el impacto que tuvo (Maldonado, comunicación personal, 13 de diciembre de 2018).

Entre otras ventajas, esta forma de trabajar permitió ganar credibilidad, pues lejos de ver competencia, la audiencia reconocía "que no era un esfuerzo por vender, era un esfuerzo por compartir información verdadera y por ayudar a la democracia del país" (Escamilla, comunicación personal, 19 de diciembre de 2018). Además, la colaboración permitió romper con barreras generadas por las burbujas de información que surgen en el contexto mediático actual. El apoyo de medios con distintos cortes editoriales permitió a Verificado 2018 alcanzar a diferentes audiencias, un aspecto importante para llevar información precisa a todo tipo de públicos. Es así que, según los participantes, la estructura colaborativa de Verificado 2018 podría tomarse en 
cuenta para proyectos futuros "para poder seguir sobreviviendo y evolucionando y adaptándose a algo nuevo que sí está demandando la gente” (González, comunicación personal, 18 de diciembre de 2018). En el contexto de crisis de credibilidad por el cual atraviesan los medios a nivel mundial y en México, esta alternativa de hacer periodismo puede servir como ejemplo para proyectos periodísticos de fact-checking o de otra naturaleza.

Creo que hay un interés, no solo de los medios por comercializar este tipo de productos sino por la gente hay una demanda, diría que hay una demanda brutal por este tipo de información verificada. No podemos negar que la gente tiene mucha desconfianza hacia los medios tradicionales, hay muchos cuestionamientos de la gente, pues sí, obviamente han habido errores, pero también hay desconfianza en general, y este tipo de proyectos devuelven la credibilidad a los medios (Larraz, comunicación personal, 18 de diciembre de 2018).

\section{Discusión}

Este trabajo analiza el proyecto de fact-checking Verificado 2018 desde la perspectiva de los periodistas que formaron parte del mismo, reconociendo la necesidad de escuchar las voces de los actores involucrados en el periodismo para construir mejores teorías acerca de este y su contribución a la democracia (George, 2013: 497). De tal forma, el presente trabajo contribuye a nuestro entendimiento sobre cómo conciben los periodistas el fact-checking y sobre cómo se desarrolla e incorpora esta práctica en el periodismo mexicano, considerando que antes de ser efectivo para informar al ciudadano, el factchecking debe establecerse dentro de una estructura (Amazeen, 2017: 1).

Los resultados de este análisis indican que los periodistas de Verificado 2018 enmarcan sus actividades de fact-checking y desmentir notas falsas como parte de las labores regulares del periodismo. Consistente con investigaciones previas (Amazeen, 2013; Cheruiyot y Ferrer-Conill, 2018: 969; Graves, 2013; Mena, 2018), los periodistas entrevistados señalaron que su misión en este proyecto era presentar información a los ciudadanos para que tomen decisiones informadas a la hora de votar en la elección presidencial. En ese sentido, hacer fact-checking no aparta al periodista de sus funciones tradicionales, como servir como vigilantes o perros guardianes, recabando y publicando información de interés para la audiencia (Christians et al., 2009), con el fin de formar a un público capaz de autogobernarse (George, 2013: 493). Es decir, como encontraron Graves et al. (2016) en el contexto estadounidense, el fact-checking apela al periodista mexicano, porque se asocia con valores de la profesión y la noción de ejercer un buen periodismo. 
No obstante, los entrevistados reconocieron que existen diferencias entre el periodismo que realizaban en Verificado 2018 y el periodismo convencional en México. El periodismo que ejecutaban en Verificado 2018, de acuerdo con sus colaboradores, requería más análisis de datos e investigación en menos tiempo, pues no se dedicaban a cubrir notas del día. Asimismo, otra diferencia importante era la relación de los periodistas con sus fuentes, de tal forma que la colaboración con estas era más horizontal y cercana. Igualmente, se fomentó una relación cercana y de lealtad con el lector, a través de redes sociales y de forma novedosa con una cuenta de WhatsApp.

Aún más importante, los entrevistados señalaron que encontraban diferencias entre el periodismo mexicano y el fact-checking llevado a cabo por Verificado 2018, debido a la mala calidad del periodismo que se hace en algunos medios en México. Los informantes atribuían estas deficiencias a la falta de personal, falta de tiempo, poca especialización. Además, eran cuidadosos de puntualizar que esta crítica no era válida para todos los medios y periodistas. Sin embargo, los colaboradores de Verificado 2018 señalaron prácticas como la "declaracionitis", la publicación de declaraciones de personajes públicos sin análisis, al considerar que esta forma de proceder implica el olvido de un elemento básico en el periodismo: corroborar la información que se publica.

Martínez-Carrillo y Tamul (2019) identificaron las estrategias de comunicación de Verificado 2018 para posicionarse como una fuente legítima de información, por ejemplo, con un manejo transparente sobre su financiamiento y metodología, asociándose con Verificado 19s (la iniciativa surgida para verificar información tras el terremoto del 19 de septiembre de 2017), e incentivando la interacción con el público. Es así que tal estudio encontró que Verificado 2018 realizó labores de reparación del campo del periodismo, mediante las cuales se busca atender una falla fundamental o amenaza en un campo ocupacional a través de nuevas alternativas, emprendiendo una reforma para proteger al periodismo (Graves y Konieczna, 2015: 1969).

Los hallazgos del presente trabajo indican que para los periodistas que trabajaron en Verificado 2018, estos riesgos para el periodismo no solo provienen de amenazas externas como la actual abundancia de noticias e información intencionalmente falsa, sino que también se debe a una falla interna en cómo se practica el periodismo en México, pues es común que se publiquen declaraciones de actores públicos sin una correspondiente verificación de la información.

En este sentido, los resultados coinciden con Cheruiyot y Ferrer-Conill (2018: 970), quienes encontraron que los periodistas que practican factchecking buscan llenar un vacío actual en el periodismo informativo, enfocándose 
en el uso de datos duros como fuente de información. En efecto, estudios previos (Reyna, 2019: 18) han señalado que el diseño del trabajo y la lógica del modelo de producción de noticias bajo el que funcionan los medios mexicanos son responsables de una descualificación que reduce a periodistas a recolectores de declaraciones, al no tener control sobre su labor. Para los periodistas colaboradores, Verificado 2018 representó una oportunidad para trabajar bajo un modelo de producción de noticias distinto y con diferentes objetivos. Ante las fallas identificadas, el periodismo mexicano apostó como alternativa por un proyecto de fact-checking, en el cual además se introdujeron formas de practicar el periodismo no convencionales, como mantener relaciones más cercanas y horizontales con sus fuentes y con el público.

$\mathrm{Al}$ reflexionar sobre las consecuencias que tuvo Verificado 2018, los entrevistados manifestaron sentirse satisfechos con el trabajo que hicieron, debido a que, desde su punto de vista, Verificado 2018 logró posicionarse como un referente al discutir información generada durante la elección, tanto ante actores políticos como ante el público. Estos logros fueron consistentes con la misión del periodismo respecto a su impacto en la democracia. Además, en estas reflexiones sobre las consecuencias del proyecto destaca el impacto que consideran que el proyecto tuvo para el periodismo mexicano.

Los periodistas subrayan el debate acerca de periodismo que surgió gracias a su labor. Como alternativa ante la situación actual del periodismo apuntalan la estructura de periodismo colaborativo que adoptó Verificado 2018. Esa forma de trabajar permitió una mejor cobertura de la elección, mayor alcance a audiencias más diversas y mayor credibilidad ante la audiencia respecto al trabajo que realizaron.

Esta experiencia puede servir en el futuro como ejemplo para proyectos periodísticos, en particular ante un escenario adverso donde medios informativos y periodismo luchan por recuperar su credibilidad y subsistir en un entorno de múltiple información y desinformación. Sin embargo, destaca que Verificado 2018 fue un proyecto considerado para operar únicamente durante la elección de ese año; es decir, este modelo de periodismo colaborativo funcionó bajo un formato temporal.

Si bien el género de fact-checking en alianza con medios tradicionales aparenta estar bien adaptado para alimentar la producción de noticias en tales medios (Graves, 2013: 261), como sucedió con el caso bajo estudio durante la elección, un periodismo colaborativo de largo plazo en México tendría que conciliar los intereses comerciales con los cuales funcionan los medios en el país y la tendencia a competir por la información con el ímpetu democrático y reformista del periodismo que incentiva a la cooperación. 
No obstante, como señalaron los periodistas de Verificado 2018, anteponer esa misión democrática del periodismo a través de la colaboración con otros medios puede ser también una estrategia para enfrentar las presiones económicas que sufren los medios en la actualidad.

Por otro lado, si bien los colaboradores de Verificado 2018 identificaban problemas en la práctica del periodismo mexicano y pusieron en acción alternativas para practicarlo, los periodistas entrevistados negaron que el objetivo del trabajo que efectuaron fuera más allá del periodismo y tuviera una misión activista. Varios de los informantes señalaron que no había un componente de activismo en la labor que realizaban, pues generar una audiencia crítica respecto a la información que se consume es parte del trabajo del periodista; es decir, tal como se ha encontrado en otros estudios (Mena, 2018), los periodistas, incluso los fact-checkers, desean mantener una distancia con el activismo.

No obstante, los entrevistados sí manifestaron en sus explicaciones que en las notas a las que denominaban como de media literacy (alfabetización mediática) había un objetivo más allá de informar y generar reflexión sobre el contenido informativo. Estas notas, sobre todo videos elaborados por el equipo de AJ Plus, se distinguían de las verificaciones de declaraciones de políticos o de noticias falsas, pues hacían referencia directamente a problemas que afectan al entorno mediático y de información, o bien instruían a los lectores sobre cómo podían cerciorarse por sí mismos de la veracidad de la información que reciben y comparten. Este contenido se alineaba con los objetivos del concepto de alfabetización mediática, que incluyen el fomento del pensamiento crítico del individuo de tal forma que entienda cómo los medios impactan en la cultura y la sociedad, y que sea capaz de identificar tendencias parciales e información incorrecta en el contenido que consume (Media Literacy Project, 2015).

Según explicaron los entrevistados, estas notas eran motivadas por un interés pedagógico y de dejar un legado en el público respecto a cómo utilizar los medios cuando Verificado 2018 terminara sus actividades. Aunque solo representaron una porción pequeña del contenido que generaron, las notas con esta intención de alfabetización mediática también puede considerarse como una estrategia alternativa mediante la cual se busca hacer una reparación del campo de periodismo, para contrarrestar la gran amenaza que representa la creación y circulación de información falsa en medios digitales. Sin embargo, destaca que aunque los periodistas reconocían el valor de este tipo de contenido, no asimilan la alfabetización mediática como una causa activista por incorporar en su labor periodística. 
En ese sentido, Graves (2013: 233) asegura que el fact-checking expone la tensión implícita en la práctica del periodismo objetivo, porque se aproxima a una retórica y objetivos reformistas. Esta tensión se manifiesta en los periodistas de Verificado 2018, quienes por un lado, no tienen problema en incorporar el fact-checking, pues se alinea con los ideales tradicionales del periodismo que apelan a los valores de su profesión, pero se rehúsan a considerar su actividad como una forma de activismo aun cuando involucre también un objetivo pedagógico que, en vista del entorno mediático inundado de desinformación, se vuelve más relevante en la actualidad.

Si bien un proyecto de fact-checking como Verificado 2018 contribuye a la discusión pública meramente con su trabajo periodístico y no tiene por qué tener una misión activista, futuros proyectos de verificación deben cuestionarse cómo los valores tradicionales del periodismo pueden limitar los alcances de su misión (Graves, 2013: 25). De igual manera, los medios y el periodismo deben reflexionar sobre su responsabilidad de participar en procesos de alfabetización mediática para, en su caso, articularla concretamente e incorporarla en su trabajo.

El presente artículo presenta dos aportaciones principales para el estudio del fact-checking en México. Primero, partiendo de trabajos anteriores (Cheruiyot y Ferrer-Conill, 2018; Martínez-Carrillo y Tamul, 2019), se establecen las estrategias utilizadas por Verificado 2018 como parte del movimiento de fact-checking mundial para reparar y reformar el periodismo: 1. Uso del fact-checking como un elemento básico del periodismo y énfasis en análisis de datos; 2 . Relaciones más horizontales con las fuentes; 3. Énfasis en la interacción con el público al cual se involucra en los procesos de verificación; 4. Periodismo colaborativo; 5. Incorporación de notas de educación mediática.

Cada una de estas estrategias marcan pauta para continuar la discusión avivada por Verificado 2018 con sus actividades durante la elección presidencial acerca de la calidad y el futuro del periodismo en México. Si bien algunas de estas estrategias están alineadas con los valores tradicionales del periodismo, otras expanden el discurso periodístico (Cheruiyot y Ferrer-Conill, 2018). En ese sentido, la segunda contribución de este trabajo es establecer en el contexto mexicano la tensión señalada por Graves (2013) y que se manifiesta en los periodistas fact-checkers de Verificado 2018 entre mantenerse dentro de los valores normativos del periodismo y el incorporar prácticas y discursos para contrarrestar el fenómeno de la desinformación y noticias falsas, los cuales se orientan más hacia una visión pedagógica y de empoderamiento del público. 
Como todo esfuerzo de investigación académica, este estudio tiene limitaciones. Los puntos de vista recolectados provienen de periodistas que se involucraron en el proyecto de Verificado 2018, por lo que la percepción del periodismo mexicano acerca de la práctica del fact-checking y la verificación no puede generalizarse a partir de estos resultados a todos los periodistas del país. Igualmente, aunque los entrevistados ofrecen sus perspectivas acerca del impacto de Verificado 2018 durante la campaña electoral, el efecto que tuvo esta labor en la opinión pública durante las elecciones no puede inferirse a partir de estas opiniones. Futuros estudios deben concentrarse en profundizar sobre tales aspectos de este fenómeno para tener una perspectiva más completa de sus alcances.

Las iniciativas de fact-checking y verificación constituyen una de las herramientas más concretas que pretenden contrarrestar el avance de fenómenos como las noticias falsas y la explosión (y explotación) de la desinformación en plataformas digitales. Luego de Verificado 2018, otros periodistas y medios han optado por desarrollar proyectos de este tipo en México, si bien con diferentes características y alcances. Este análisis sobre la experiencia de Verificado 2018 apunta que los periodistas reconocen fallas y amenazas en la práctica del periodismo en el país y están dispuestos a apostar por alternativas que contribuyan a mejorar la profesión, por lo que sirve como una exploración respecto al potencial y posibles retos para estos proyectos en el futuro.

\section{Referencias}

Allcott, Hunt y Gentzkow, Matthew (2017), "Social media and fake news in the 2016 election", en Journal of Economic Perspectives, vol. 31, núm. 2, Estados Unidos: American Economic Association.

Amazeen, Michelle (2013), "Making a difference: A critical assessment of fact-Checking in 2012, en Media Policy Initiative Research Paper, Estados Unidos: New America Foundation.

Amazeen, Michelle (2015), "Sometimes political fact-checking works. Sometimes it doesn't: Here's what can make the difference", en The Washington Post, Disponible en: https://www.washingtonpost.com/news/monkey-cage/wp/2015/06/03/sometimespolitical-fact-checking-works-sometimes-it-doesnt-heres-what-can-make-thedifference [25 de junio de 2019].

Amazeen, Michelle (2017), "Journalistic interventions: The structural factors affecting the global emergence of fact-Checking", en Journalism, vol. 21, núm. 1, Estados Unidos: Sage.

Berger, Arthur (2000), Media and Communication Research Methods, Inglaterra: Sage.

Brandtzaeg, Petter et al. (2018), "How Journalists and Social Media Users Perceive Online Fact-Checking and Verification Services”, en Journalism Practice, vol. 12, núm. 9, Inglaterra: Routledge. 
Boyatzis, Richard (1998), Transforming qualitative information: Thematic analysis and code development, Estados Unidos: Sage.

Cheruiyot, David y Ferrer-Conill, Raul (2018), "Fact-Checking Africa", en Digital Journalism, vol. 6, núm. 8, Inglaterra: Taylor \& Francis.

Christians, Clifford et al. (2009), Normative theories of the media: Journalism in democratic societies, Estados Unidos: University of Illinois Press.

De la Torre, Karen (2018), "Los falsos lazos familiares entre Ricardo Anaya y los Salinas", en Verificado 2018. Disponible en https://verificado.mx/los-lazos-falsos-entre-anayay-los-salinas/ [20 de diciembre de 2019].

Díaz, Bertha (2018), "Inexactos, los cálculos de López Obrador para medir la penetración de la telefonía móvil”, en Verificado 2018. Disponible en: https://verificado.mx/ inexactos-calculos-amlo-telefonia-movil/ [20 de diciembre de 2019].

Echeverría, Borja (2017), "Más 'fact-checking' contra la posverdad", en Cuaderno de Periodistas, núm. 33, España: Asociación de la Prensa de Madrid. Disponible en: http://www.cuadernosdeperiodistas.com/mas-fact-checking-la-posverdad/ [26 de abril de 2018].

Edelman Trust Barometer (2018), "2018 Edelman Trust Barometer Global Report. Edelman”. Disponible en: https://www.edelman.com/trust-barometer [25 de abril de 2018].

Flueckiger, Simone (2019), "Verificado 2018: Fighting missinformation collaboratively", en World Association of Newspapers and Newspublishers. Disponible en: https://blog.wanifra.org/2019/11/12/verificado-2018-fighting-misinformation-collaboratively [20 de diciembre de 2019].

Fridkin, Kim et al. (2015), "Liar, liar, pants on fire: How fact-checking influences citizens' reactions to negative advertising”, en Political Communication, vol. 32, núm. 1, Estados Unidos: Taylor \& Francis.

George, Cherian (2013), "Diversity around a democratic core: The universal and the particular in journalism”, en Journalism, vol. 14, núm. 4, Estados Unidos: Sage.

Glaser, Barney y Strauss, Anselm (1967), The Discovery of grounded theory: strategies for qualitative research, Estados Unidos: Aldine Publishing Company.

Gottfried, Jeffrey et al. (2013), "Did fact checking matter in the 2012 presidential campaign?”, en American Behavioral Scientist, vol. 57, núm. 11, Estados Unidos: Sage.

Graves, Lucas (2013), "Deciding what's true: Fact-checking journalism and the new ecology of news" [tesis doctoral], Estados Unidos: Columbia University.

Graves, Lucas (2018), "Boundaries Not Drawn: Mapping the institutional roots of the global fact-checking movement", en Journalism Studies, vol. 19, núm. 5, Inglaterra: Routledge.

Graves, Lucas et al. (2016), "Understanding Innovations in Journalistic Practice: A Field Experiment Examining Motivations for Fact-Checking”, en Journal of Communication, vol. 66, núm. 1, Estados Unidos: International Communication Association.

Graves, Lucas y Konieczna, Magda (2015), "Sharing the News: Journalistic Collaboration as Field Repair", en International Journal of Communication, vol. 9, Estados Unidos: International Communication Association. Disponible en: https://ijoc.org/index. $\mathrm{php/ijoc/article/viewFile/3381/1412} \mathrm{[20} \mathrm{de} \mathrm{junio} \mathrm{de} \mathrm{2019].}$

Khatib, Dima y Larrea, Diana (2018), “Bienvenid@s a \#Verificado2018!”, en Verificado 2018. Disponible en: https://verificado.mx/bienvenidos-a-verificado-2018/ [20 de junio de 2019]. 
Kuklinski, James et al. (2000), "Misinformation and the currency of democratic citizenship", en The Journal of Politics, vol. 62, núm. 3, Estados Unidos: The University of Chicago Press.

López, Xosé et al. (2016), "El fact checking como reclamo y como servicio en los cibermedios", en Revista Telos, núm. 103, España: Fundación Telefónica.

Magallón, Raúl (2019), "Verificado México 2018. Desinformación y fact-checking en campaña electoral”, en Revista de Comunicación, vol. 18, núm. 1, Perú: Universidad de Piura. DOI: https://doi.org/10.26441/RC18.1-2019-A12 Disponible en: https:// revistadecomunicacion.com/article/view/1034 [20 de diciembre de 2019].

Martínez, Marcos (2018), "México: Los 'bots', 'trolls' y otros trucos de manipulación en internet que amenazan las próximas elecciones presidenciales”, en $B B C$. Disponible en: https://www.bbc.com/mundo/noticias-america-latina-44302996 [20 de junio de 2019].

Martínez-Carrillo, Nadiay Tamul,Daniel(2019), “(Re)constructingProfessional Journalistic Practice in Mexico: Verificado's Marketing of Legitimacy, Collaboration, and Pop Culture in Fact-Checking the 2018 Elections", en International Journal of Communication, vol. 13, Estados Unidos: International Communication Association. Disponible en: https://ijoc.org/index.php/ijoc/article/view/10933 [20 de junio de 2019].

Media Literacy Project (2015), “What is media Literacy?", en Media Literacy Project. Disponible en: http://medialiteracyproject.org/learn/media-literacy/ [25 de abril de 2018].

Mena, Paul (2018), "Principles and boundaries of fact-checking: Journalists' perceptions", en Journalism Practice, vol. 13, núm. 6, Inglaterra: Routledge.

Muñoz-Torres, Juan Ramón (2012), "Truth and objectivity in journalism: Anatomy of an endless missunderstanding”, en Journalism Studies, vol. 1, núm. 4, Inglaterra: Routledge.

Patton, Michael (1990), Qualitative evaluation and research methods, Estados Unidos: Sage. Pingree, Raymond et al. (2018), "Checking facts and fighting back: Why journalists should defend their profession”, en PLoS ONE, vol. 13, año 12, Estados Unidos: Plos.

Potter, James (2010), “The State of Media Literacy”, en Journal of Broadcasting \& Electronic Media, vol. 54, núm. 4, Inglaterra: Routledge.

Redacción Animal Político (2015), "Famosos hacen propaganda a favor del Partido Verde, a un día de la elección”, en Animal Político. Disponible en: https://www.animalpolitico. com/2015/06/famosos-hacen-propaganda-a-favor-del-partido-verde-a-un-dia-de-laeleccion/ [20 de junio de 2019].

Reyna, Víctor (2019), "De la estandarización a la descualificación: las consecuencias indeseadas de la modernización del periodismo mexicano”, en Comunicación y Sociedad, año 16, México: Universidad de Guadalajara.

Verificado 2018 (2018a), “¿Qué es Verificado 2018? Verificado 2018”, en Verificado 2018. Disponible en: verificado.mx/que-es-verificado-2018/ [26 de abril de 2018].

Verificado 2018 (2018b), "Hasta Luego, Verificado 2018”, en Verificado 2018. Disponible en https://verificado.mx/hasta-luego-hoy-cierra-verificado-2018/ [20 junio de 2019].

Vizoso, Ángel y Vázquez-Herrero, Jorge (2019), "Plataformas de fact-checking en español. Características, organización y método”, en Communication \& Society, vol. 32, núm. 1, España: Universidad de Navarra.

Walter, Nathan y Murphy, Sheila (2018), "How to unring the bell: A meta-analytic approach to correction of misinformation”, en Communication Monographs, vol. 85, núm. 3, Estados Unidos: National Communication Association.

Wintersieck, Amanda (2017), "Debating the truth: The impact of fact-checking during electoral debates", en American Politics Research, vol. 45, núm. 2, Estados Unidos: Fordham University. 
Anexo

\section{Tabla 1}

Nombres de entrevistados y sus roles en Verificado 2018

\begin{tabular}{cll}
\hline Entrevista & Nombre & Rol en Verificado 2018 \\
\hline 1. & Ángel Plascencia & Reportero \\
\hline 2. & Syndy García & $\begin{array}{l}\text { Reportera de medio afiliado a Verificado } \\
2018\end{array}$ \\
\hline 3. & Tania Montalvo & Coordinadora \\
\hline 4. & Montserrat Maldonado & Reportera \\
\hline 5. & Yuriria Ávila & Analista de datos y reportera \\
\hline 6. & Oscar González & Analista de datos \\
\hline 7. & Irene Larraz & Reportera \\
\hline 8. & María José López & Community Manager \\
\hline 9. & Santiago Escamilla & Community Manager para Whatsapp \\
\hline 10. & Adolfo García & Community Manager \\
\hline 11. & Ana Karen de la Torre & Reportera \\
\hline 12. & Diego de la Mora & Coordinador de metodología y datos \\
\hline 13. & Aldo Nicolai & Reportero \\
\hline
\end{tabular}

Fuente: Elaboración propia.

Rocío Galarza Molina. Doctora en Comunicación por la Universidad de Missouri. Labora en la Facultad de Ciencias Políticas y Relaciones Internacionales, de la Universidad Autónoma de Nuevo León. Líneas de investigación: comunicación política, deliberación en línea, rol de medios de información en democracias en transición. Publicaciones recientes: Galarza Molina, Rocío y Jennings, Fred (2017), "The role of civility and metacommunication on Facebook discussions", en Communication Studies, vol. 00, núm. 00, Estados Unidos: Asociación de Comunicación de Estados Centrales; Warner, Ben et al. (2017), "Comic agonism in the 2016 campaign: A study of Iowa Caucus rallies”, en American Behavioral Scientist, Estados Unidos: Sage Publishing; Warner, Ben et al. (2017), "The effects of debate viewing on candidate image perceptions in the 2016 televised presidential general election debates", en Hinck, Edward [ed.], Presidential debates in a changing media environment, Estados Unidos: Praeger. 